\title{
Do primary care placements influence career choice: what is the evidence?
}

\author{
Key word: undergraduate; placement; longitudinal; general practice; 10-point plan; \\ https://www.hee.nhs.uk/sites/default/files/documents/WES_The-future-of-primary-care.pdf
}

\section{Background}

General practice (GP) is a fundamental element of the UK National Health Service(NHS) healthcare model [1]. Pressures on the system as a result of demographic change, increasing patient complexity and bureaucratic demands are inexorably rising. The primary care workforce must both adapt and expand. However fewer medical graduates are choosing GP as a career, and successive recruitment rounds in recent years have failed to fill UK GP training posts [2,3]. The Department of Health in England aims to recruit 50\% of medical graduates into GP. However, the 2016 Career Destination Report shows that only $20 \%$ of graduates intend to pursue a career in GP[4]. The shortfall is further exacerbated by an ageing workforce that is approaching retirement, and significant numbers of younger general practitioners driven away or reducing their clinical commitment by increased workload and decreased job satisfaction[5]. In response, Health Education England (HEE) and the Medical Schools' Council (MSC) led a taskforce to explore the factors that influence medical students when considering a career in GP. Their recommendations were published in the 2016 Wass Report, "By Choice - not by Chance" [6]. The paper presented below is based on the original literature review which underpinned the Wass report, and aims to explore further a key area addressed by the report i.e. the influence of medical school primary care placements on student career choice.

\section{The international evidence}

A large component of undergraduate (UG) medical education is delivered through clinical placements. In the UK placements are spread across different specialities and are predominantly hospital based. Each placement addresses the curriculum but also gives students a flavour of that specialty to inform their possible future career choices. One multi-country systematic review by Verma et al (2016) found that students who had more primary care focused placements were more likely to pursue GP [7]. Another international review also found positive influences from more GP UG experience [8]. This review however distinguished between traditional block placements (TBP) and longitudinal GP placements. Studies on TBP found students had a positive intention towards a GP career, which was most pronounced immediately after the placements, but these studies did not assess career destination. Studies on longitudinal placements assessed career destination and found a positive association. The majority of longitudinal programmes (undefined) were additional extended primary care placements, while others delivered a longitudinal experience by adapting the curriculum to be primary care focused. Programme formats varied in style, duration and frequency. Other evidence suggests that the longer the duration of a placement, the greater the impact [9]. In 
one US longitudinal programme, students felt better integrated and that they could have more meaningful "hands-on experience" [10], a view supported by another systematic review [11]. There may be selection bias with longitudinal programmes since most such programmes (19 out 21) recruited students interested in primary care, and the effect of this self-selection could not be disconnected from the direct impact of the longitudinal programme[8]. Of the two programmes that controlled for selection bias, one found a direct significant association on career intention, whereas the other found the effect of the pre-existing interest to be a stronger association.

An international narrative review compared longitudinal style placements with TBPs and was not just limited to primary care placements (see table 1) [9]. It used the term 'longitudinal integrated clerkships' (LIC) and defined it according to the International Consortium of Longitudinal Integrated Clerkships criteria [9]. The minimum length to achieve a longitudinal experience remains contentious and not all programmes deliver an truly integrated curriculum [9]. Only programmes that were longer than six months and incorporated an integrated curriculum were included in this review. This study again demonstrated that longitudinal primary care programmes positively influence towards a career in primary care and highlighted the influence of role models and career mentorship. Although initially introduced in rural areas to promote students towards primary care, longitudinal integrated programmes are now also offered in urban areas and other specialties [9]. Longitudinal programmes are found to promote a number of characteristics including clinical responsibility, developing a broad skillset, patient-centred practice and holistic practice)[9] which can all complement primary care practice. Another feature of longitudinal programmes is the reduced exposure to potentially negative effects of institutional hidden curricula [12]. Furthermore, longitudinal programmes claim to offer a nurturing environment by placing greater focus on strategies to deal with the challenges of primary care. The rural Minnesota programme provided a better training experience through authentic practice, mentoring, one-to-one teaching and putting students in education networks with other students [10]. Students felt the optimised placement experience made them feel better prepared for a career in GP. Some programmes were designed in a way to target specific challenges. The Broken Hill longitudinal programme enabled interprofessional learning with students from other healthcare disciplines and placed the students in shared accommodation, which led to greater appreciation of the allied healthcare professionals and recognition of the need for a multidisciplinary team in the community [13]. The multidisciplinary network may have also reduced the sense of isolation that some students experience in primary care. 
Table 1 International Literature: longitudinal Integrated programmes as compared to Traditional Block Placements

\begin{tabular}{|l|l|}
\hline \multicolumn{1}{|c|}{ Positives } & \multicolumn{1}{c|}{ Negatives } \\
\hline Students are more likely to pursue GP [9] & $\begin{array}{l}\text { Learning can be unpredictable, particularly in } \\
\text { primary care - dependent on patient } \\
\text { presentation[9] }\end{array}$ \\
\hline Academic results mostly comparable or better[9] & $\begin{array}{l}\text { Students find it difficult to link their learning } \\
\text { against the curriculum and assessments[9] }\end{array}$ \\
\hline Suitable for integrated curricula[9] & \\
\hline More patient-centred[9] & \\
\hline $\begin{array}{l}\text { Longer placement allows students to develop } \\
\text { and have a meaningful clinical experience[11] }\end{array}$ & \\
\hline Student contribute more to service provision[9] & \\
\hline Greater exposure to medical ethics[9] & \\
\hline $\begin{array}{l}\text { Reduced exposure to institutional hidden } \\
\text { curriculum[12] }\end{array}$ & \\
\hline
\end{tabular}

\section{The UK context}

The majority of international research in this area has focused on rural settings in the USA and Australia, born out of concerns around rural GP shortages. The UK literature on UG primary care placements is limited but there are some consistencies in the findings. The international evidence demonstrates a link between doctors pursuing GP and the amount of UG GP placement experience. Recently, for the first time, this was also shown in the UK [14]. The quality of placements is also important. A focus group study of students from nine UK medical schools identified common findings with the international literature [15]. Students explained that they are more likely to pursue GP if their placements were good quality, provided authentic practice and showed the impact which GP can make. Students were heavily influenced by GP tutors through role-modelling. There were some fears raised around the potential feeling of isolation in GP and the sense that the job is just too difficult. In a qualitative study of two UK medical schools, students again stated the importance of the quality of training, hands-on experience and powerful influence of GP tutors[16]. Here students reported that longer placements were educationally more valuable. Participants also explained that primary care was a more supportive environment to develop the core competencies of clinical medicine and wider aspects of healthcare, which suggests that integrated curricula based in the community can work in the UK. Interestingly a study in Ireland found that an integrated curriculum was well received, moreover the integrated curriculum was better received when it was based in primary care [17]. 


\section{Implications- How to improve UK undergraduate primary care placements in GP}

The current format of UK medical training is not producing enough GPs. Currently only $13 \%$ of UK UG medical education is delivered in primary care [18]; if high quality sustained UG exposure in GP is key in GP career choice then it could be argued this volume is insufficient to meet UK health objectives. Healthcare is increasingly provided in the community and mainly led by GPs [1], however UG medical education in the UK is not reflecting this change. A systematic review of medical education in the UK GP setting, showed that a wide range of learning opportunities is available [19]. However, it also showed how positive and negative attitudes towards GP can shape students' learning experiences in this socio-cultural learning space (e.g. placements not being valued if perceived not to align with UG assessments; text book learning etc.). Primary care placements should be of good quality and strategically structured to ensure a range of UG exposure throughout the curriculum. Good quality early exposure can address negative preconceptions that can be found in the 'hidden-curriculum' [20]. One aspect of the hidden curriculum is the phenomenon of subtly discouraging students from primary care through institutional cultures (e.g. specialty hierarchy and badmouthing)[21]. Primary care placements or assistantships taking place in later stages of the course can also capitalise on the fact that students' intention to pursue GP is most pronounced after a primary care placement [8]. Furthermore, a primary care placement in the final year means students will be capable of more teamwork, hands-on involvement and clinical responsibility, which are important for their learning and enjoyment of primary care $[10,15,16]$.

GP is often described as the most important specialty in the NHS [1]. There are however several misconceptions among medical students about GP [22]. For instance, many students perceive it as not intellectually stimulating [22], when in fact GPs are the experts in generalism and multicomorbidity and so constantly face clinical dilemmas across several specialities simultaneously. GP also offers great opportunities for academic endeavour through teaching and research. UG teaching and placements must reflect this reality to ensure students are not put off by misconceptions. In addition to providing positive role models this can be achieved via an integrated curriculum where students meet competencies of other specialities in their community placements, which will provide a more realistic experience of primary care and allow students to see the sort of impact that GPs make within the NHS. For example studies have shown that GPs managing end of life care in the community were inspirational to students [15], and when health disabilities were taught in the community students enjoyed it and expressed a desire for more clinical time in the community [23]. Integrated curricula based in primary care are more representative of the community based healthcare model that the NHS is moving towards. Such curricula would also enable a longitudinal approach, which will allow students to integrate into practices and experience the wider aspects of community healthcare. Longer placements would allow students to gain a balanced view of population health care needs and gain first-hand experience of the work and the rewards of GP [10]. Suggestions were also made that the generalism in primary care is better aligned with students' UG learning needs compared to the specialised approach in hospitals [17]. Another argument for students to be based in primary care is because they feel safer and better supported, and prefer GPs as supervisors $[16,17]$.

Primary care placements should be designed in a way to minimise isolation. GPs take on fewer medical students and some students have a poor experience due to perceived isolation, especially when the placement is far from the central campus [24]. This can be improved by training students 
from other disciplines in the community in parallel [13]. Inter-professional learning and living creates positive educational networks and improves the appreciation of the multidisciplinary team [13]. It also aligns primary care placements with the multidisciplinary vision outlined for GP in the Roland Report [25]. This report on the future of GP, describes the need for large primary care centres with better infrastructure and greater multidisciplinary presence to support GPs [25]. Implementing this may be central to countering the common perception among students that GPs are professionally isolated, which negatively affects their career aspirations [15]. The current widespread negativity among GPs themselves as a result of their heavy workloads and underresourced services is also affecting students [1]. Enthusiastic GP role models are a positive influence on students' perception of primary care, and observing demoralised GPs who are not enjoying their work may discourage students $[15,16]$. It is easy to say we should encourage GPs to present a more positive picture, but GPs are not actors and addressing the circumstances causing the negativity is crucially important.

\section{Conclusion}

Evidence shows that medical students can be encouraged to pursue GP as a career if they undertake community placements which are of sufficient quantity, quality and duration. Efforts to maximise positive perceptions of primary care are needed not only undergraduate level but also within NHS organisational culture and policy. If we are to approach the required number of medical graduates entering GP, undergraduate community placements must provide consistently good experience, thereby encouraging students to form positive perceptions of a potential career in primary care. Evidence based optimisation of undergraduate primary care placements is urgently needed in the UK, but will only be possible if these changes are well integrated into the organisation and delivery of UG medical education and vitally, if the job of NHS GP becomes more attractive. These developments require adequate investment of resources to allow GPs the time and space to provide sufficient number and quality of placements in order to enthuse the next generation of doctors. Meanwhile more high quality research is undoubtedly needed to help us understand better the complexities of medical student career choice. 


\section{References}

1. General Practice Forward View. NHS England. 2016.

2. Specialty recruitment: round 1 - acceptance and fill rate. Health Education England 2017.

3. Rimmer, A., GP recruitment opens for third round. BMJ Careers 2015.

4. The Foundation Programme: Career Destination Report 2016. The Foundation Programme. 2016.

5. Securing the Future GP Workforce Delivering the Mandate on GP Expansion: GP Taskforce Final Report. NHS. 2014.

6. By choice - not by chance: Supporting medical students towards future careers in general practice. Health Education England. 2016.

7. Verma, P., et al., A systematic review of strategies to recruit and retain primary care doctors. BMC Health Serv Res, 2016. 16: p. 126.

8. Pfarrwaller, E., et al., Impact of Interventions to Increase the Proportion of Medical Students Choosing a Primary Care Career: A Systematic Review. J Gen Intern Med, 2015. 30(9): p. 1349-58.

9. Walters, L., et al., Outcomes of longitudinal integrated clinical placements for students, clinicians and society. Med Educ, 2012. 46(11): p. 1028-41.

10. Halaas, G.W., et al., Recruitment and retention of rural physicians: outcomes from the rural physician associate program of Minnesota. J Rural Health, 2008. 24(4): p. 345-52.

11. Crampton, P.E., J.C. McLachlan, and J.C. Illing, A systematic literature review of undergraduate clinical placements in underserved areas. Med Educ, 2013. 47(10): p. 969-78.

12. Hirsh, D., et al., Educational outcomes of the Harvard Medical School-Cambridge integrated clerkship: a way forward for medical education. Acad Med, 2012. 87(5): p. 643-50.

13. Roberts, C., et al., A longitudinal integrated placement and medical students' intentions to practise rurally. Med Educ, 2012. 46(2): p. 179-91.

14. Alberti, H., et al., Exposure of undergraduates to authentic GP teaching and subsequent entry to GP training: a quantitative study of UK medical schools. Br J Gen Pract, 2017. 67(657): p. e248-e252.

15. Nicholson, S., A.M. Hastings, and R.K. McKinley, Influences on students' career decisions concerning general practice: a focus group study. Br J Gen Pract, 2016. 66(651): p. e768-75.

16. Newbronner, E., et al., Creating better doctors: exploring the value of learning medicine in primary care. Educ Prim Care, 2017. 28(4): p. 201-209.

17. Kelly, M., D. Bennett, and S. O'Flynn, General practice: the DREEM attachment? Comparing the educational environment of hospital and general practice placements. Educ Prim Care, 2012. 23(1): p. 34-40.

18. Harding, A., et al., Provision of medical student teaching in UK general practices: a crosssectional questionnaire study. Br J Gen Pract, 2015. 65(635): p. e409-17.

19. Park, S., et al., A BEME systematic review of UK undergraduate medical education in the general practice setting: BEME Guide No. 32. Med Teach, 2015: p. 1-20.

20. Firth, A. and V. Wass, Medical students' perceptions of primary care: the influence of tutors, peers and the curriculum. Education for Primary Care, 2007. 18(3): p. 364-72.

21. Ajaz, A., et al., BASH: badmouthing, attitudes and stigmatisation in healthcare as experienced by medical students. BJPsych Bull, 2016. 40(2): p. 97-102.

22. Cleland, J.A., et al., A survey of factors influencing career preference in new-entrant and exiting medical students from four UK medical schools. BMC Med Educ, 2014. 14: p. 151.

23. Watmough, S., P. Leftwick, and S. Alexander-White, An evaluation of medical students' views on the introduction of a community placement and its impact on their understanding of patients with disabilities. Educ Prim Care, 2014. 25(1): p. 36-42.

24. Bartlett, M., et al., Teaching undergraduate students in rural general practice: an evaluation of a new rural campus in England. Rural Remote Health, 2016. 16(2): p. 3694. 
25. The future of primary care: Creating teams for tomorrow. Report by the Primary Care Workforce Commission. 2015. 